\title{
Le fonti e le tematiche teologiche della Regula di Eugippio
}

Le notizie storiche precise su Eugippio sono purtroppo scarse. L'identificazione relativamente recente del codice manoscritto Parisinus Latinus 12634, ridenominato Codex E, fornì qualche tessello in più ad un mosaico ancora incompiuto, ma soprattutto contribuì a restituire ad Eugippio uno spazio più ampio negli studi letterari e monastici². Il suo stesso nome ci è giunto tramandato in più versioni ortografiche: Euepius, Eugepius, Eugipius, Eugyppius ed Eugippius. L'ultimo, ormai codificato ufficialmente, è quello che compare negli scritti dei suoi insigni amici: Cassiodoro, ministro del re ostrogoto Teodorico e poi fondatore del Vivarium, il vescovo Fulgenzio di Ruspe e il diacono Ferrando di Cartagine ${ }^{3}$.

1 Prof. dr. hab. Bazyli Degórski O.S.P.P.E., professore di patrologia e di teologia dogmatica presso la Facoltà di Teologia della Pontificia Università S. Tommaso d'Aquino a Roma, e professore di patrologia presso la Pontificia Facoltà Teologica "Teresianum" a Roma; e-mail: osppe.roma@gmail.com; ORCID: 0000-0003-1997-2769.

2 Cf. B. Degórski - L. Mirri, Eugippio. La Regola, Collana di Testi Patristici 183, Roma 2005, p. 5.

3 Cf. Cassiodorus, De institutione divinarum et saecularium litterarum 23; Fulgentius Ruspensis, Epistula 5; Ferrandus Carthaginensis, Epistula 4. Cf. Storia della Chiesa, ed. H. Jedin, , Già e non ancora 3, Milano 1988, p. 226 (il diacono Ferrando e la Chiesa nordafricana), 317-326 (Eugippio e il monachesimo latino in Italia nei secc. V e VI), 338-353 (Fulgenzio di Ruspe e le dispute dogmatiche tra cattolici e ariani), 372-390 (Isidoro di Siviglia e Cassiodoro); H. Leclercq, Eugippius, DACL V 702-704; G. de Plinval, Eugippius, DSp IV 1684-1685; M. Cappuyns, Eugippius, DHGE XV 1376-1378; R. Noll, Eugippius. Das Leben des heiligen Severin, Berlin 1963, p. 13-34; 
Eugippio "scripsit et regulam monachis consistentibus in monasterio sancti Severini, quam eisdem moriens quasi testamentario iure reliquit"4. Questa notizia lasciata da sant'Isidoro di Siviglia trattando, tra gli altri personaggi illustri, dell'abate di Lucullano (oggi Pizzofalcone, presso Napoli), è rimasta fino alla seconda metà del secolo XX l'unica testimonianza sull'esistenza di una Regula monastica attribuita ad Eugippio, più conosciuto come autore della Vita S. Severini ${ }^{5}$.

Conservata è pure un'altra opera di Eugippio, gli Excerpta ex operibus S. Augustini, citati da Cassiodoro e dedicati dall'autore alla vergine Proba ${ }^{6}$. Pertanto, quasi per quindici secoli la Regula di Eugippio fu ritenuta perduta.

Ad opera di H. Vanderhoven di F. Masai e di P.B. Corbett venne pubblicata, nel 1953, un'edizione diplomatica dei due più antichi codici manoscritti contenenti l'anonima Regula Magistri: i codici parigini 12205 (del VII secolo ${ }^{7}$ ) e 12634. Il secondo di essi, utilizzato per un estratto del medesimo documento, va invece ritenuto già del VI secolo. Inoltre, il suo ambiente culturale di provenienza è indicato nell'Italia centro-meridionale ${ }^{8}$.

Stilando l'edizione critica della Regula Magistri vent'anni dopo, sulla base di questi due interessanti codici, Adalberto de Vogüé, O.S.B., concentrò la propria attenzione sul manoscritto Parisinus Latinus 12643, corrispondente alla seconda parte del codice, contenuta nei fogli $9^{\mathrm{r}}-7^{\mathrm{v}}$ della sua edizione. Oltre a un' estrapolazione della Regula Magistri, l'an-

M. Buedinger, Eugippius. Eine Untersuchung, "Sitzungsberichte der Österreichischen Akademie der Wissenschaften in Wien" 91 (1978) p. 793-814.

4 Isidorus Hispalensis, De viris illustribus 26, 34, PL 83, 1097.

5 Cf. Eugippius, Vita S. Severini, CSEL 9/2 e SCh 374.

6 Cf. Eugippius, Epistula ad Probam, CSEL 9/1, 1-4; Cassiodorus, De institutione divinarum litterarum 23, PL 70, 1105-1150.

7 Cf. G. Turbessi, Regole monastiche antiche, Roma 1978, p. 367.

8 Cf. A. Genestout, Le plus ancien témoin manuscrit de la Règle du Maître: le Parisinus latin 12634, "Scriptorium" 1 (1946/47) p. 129-142; J. Leclercq, Autor d'un manuscrit de la Règle du Maître, RBen 57 (1947) p. 210-212. 
tico documento presentava infatti un florilegio di diversi altri brani estratti dalla letteratura monastica cristiana orientale ed occidentale e composti a modo di una vera e propria Regula, non disdegnando all'occorrenza originali mutazioni rispetto a quelle che andavano dunque considerate piuttosto "fonti", che non mere "matrici" poi ricopiate. Vi si poteva scorgere, nell'insieme, non una somma di frammenti trascritti, bensì una delicata e quasi impercettibile opera di rielaborazione di testi abbinati secondo un criterio ben preciso e la finalità di un "libello" a se stante.

Ma a chi si doveva attribuire l'abile redazione di tale centone? Eccetto il brano d'esordio del documento, esplicitamente attribuito a sant'Agostino, tutti gli altri riportati risultavano anonimi, benché di facile identificazione per autore oppure opera. A. de Vogüé notò che quanto contraddistingueva ogni frammento in suo inizio era la lettera "E", citata una volta soltanto però come lettera iniziale per la parola Excerptum, "estratto" appunto. Gli parve allora quello un indizio sufficiente ed illuminante per avanzare l'ipotesi che quella "E" inviasse all'iniziale del nome dell'autore dell'anonima composizione letteraria monastica del codice Parisinus Latinus 12634, Eugippio, e di essere pertanto davanti a quella Regula segnalata da sant' Isidoro di Siviglia come una sorta di testamento spirituale dell'abate di Lucullano ai monaci di San Severino, non molto tempo prima di morire, circa tra il 530 e il $535^{9}$.

La prima analisi cui procedette, quindi, fu quella di critica del testo nel raffronto con entrambe le opere di Eugippio conservate: gli Excerpta ex operibus $S$. Augustini e la Vita S. Severini ${ }^{10}$. La prima opera di Eugippio è quella che, innanzi tutto, ha peculiarità identica: essa pure, come la Regula, è un florilegio. Tra i due componimenti, vi è ampia differenza di lunghezza: 1'una, gli Excerpta, constavano originalmente di 338 capitoli $^{11}$, l'altra, la Regula, di 46. Tuttavia, la loro somiglianza è notevole per

\footnotetext{
Cf. Degórski - Mirri, Eugippio. La Regola, p. 6-7.

10 A. de Vogüé, La Règle d'Eugippe retrovée?, RAM 47 (1971) p. 233-265.

11 Cf. P. Siniscalco, Il numero primitivo degli Excerpta di Eugippius, REAug 10 (1964) p. 331-342.
} 
genere e, subito, pure per esordio. Entrambi gli scritti, infatti, iniziano con un testo di sant'Agostino: l'uno con una lettera del Vescovo di Ippona a san Girolamo, la 147 che tratta dei costumi ecclesiastici, l'altro con la Regula S. Augustini. Entrambi i testi selezionati da Eugippio, poi, sono presentati interamente, e non a brani, e trattano dell'amore fraterno, tessendone l'elogio evangelico.

Circa la Regula attribuita a sant'Agostino, si tratta dell'Ordo Monasterii unito al Praeceptum ${ }^{12}$, testi nei quali si proclama la carità unificante la comunità secondo l'osservanza del comandamento dell'amore a Dio e al prossimo. Corrispondenza tra i due documenti è pure nella loro conclusione, dove convergono per contenuto, cioè l'amore fondamento della vita comune, a motivo dei due estratti da san Basilio e san Girolamo riportati a fine della Regula. In parallelo, gli Excerpta terminano con due capitoli agostiniani di medesima tematica ${ }^{13}$.

Analogie si riscontrano anche nel modo di connettere capitoli o brani tra loro: in entrambi i lavori spesso sono omesse frasi o intere pagine centrali del testo ricopiato. Vi è dunque un medesimo criterio di selezione e di cerniera degli estratti: privilegiato è l'argomento, e sotto la stessa intitolazione è possibile riscontrare frammenti di opere o di autori diversi, perché di identico contenuto. Infine, tra le altre affinità, simile in entrambi gli scritti è persino il modo di abbreviare i testi latini delle fonti ed il metodo redazionale in genere. Si riscontrano gruppi di brani a tema comune, sequenze tematiche abbinate a sequenze locali, costituite queste ultime dallo scorrere continuativamente un'opera estraendone via via i paragrafi interessati alla tematica in questione. Questo procedimento molto particolare, da Eugippio è adottato per redigere il florilegio

12 Cf. G. Lawless, An Overview of Augustine's Monasticism and some Suggestions for Further Research, in: The Spirituality of Ancient Monasticism. Acts of the International Colloquium held in Cracow-Tyniec 16-19 $9^{\text {th }}$ November 1994. Specialized Contributions, ed. M. Starowieyski, Pontificia Academia Theologica Cracoviensis, Facultas Theologica. Studia 4/1, Tyniec - Kraków 1995, p. 135-161.

13 Cf. Degórski - Mirri, Eugippio. La Regola, p. 7-8. 
di estratti dalle opere di sant'Agostino e uguale si scopre essere nella compilazione della Regula del codice Parisinus Latinus 12634. Ulteriore particolarità è che, chi ha composto la Regula ha pure, come Eugippio negli Excerpta, un proprio metodo personale nel procedere per sequenza progressiva, e cioè il tornare indietro anche a metà di una sequenza locale e di lì ripartire improvvisamente con una nuova serie progressiva ${ }^{14}$.

Tutto quanto evidenziato come significativo, da un'analisi condotta da A. de Vogüé anche su diversi altri dettagli pure filologici, consolida la tesi dell'identità di autore tra i due documenti: Eugippio ${ }^{15}$.

Il confronto della Regula con la Vita S. Severini riscontra minor somiglianza per il fatto in sé della diversità di genere letterario. Le affinità più evidenti sono comunque importanti: conformità linguistica e stilistica, contenutistica sul valore della preghiera incessante e della vita comune. Vi è inoltre la strana corrispondenza tra i 46 capitoli nei quali Eugippio articola la Vita S. Severini e i 46 estratti di autori diversi con i quali il redattore del codice 12634 - rinominato Codex $E$ - ha composto il centone da lasciare in eredità spirituale ai monaci congregati nel monastero di san Severino, come informa appunto sant'Isidoro di Siviglia. Coincidenza fortuita oppure, perseguendo uno schema logico di continuità e d'interpretazione di un certo ideale ascetico, maturato dal carisma del fondatore nell'esperienza dei suoi discepoli, intendimento preciso voluto da Eugippio? ${ }^{16}$

14 Cf. Degórski - Mirri, Eugippio. La Regola, p. 8.

15 Cf. M.M. Gorman, The Manuscript Tradition of Eugippius, "Excerpta ex operibus sancti Augustini”, RBen 92 (1982) p. 7-32, 229-265.

16 Cf. A. de Vogüé, Quelques observations nouvelles sur la Règle d'Eugippe, "Benedictina" 22 (1975) p. 31-41: l'autore qui presenta la nuova divisione in capitoli e versetti da lui apposta al testo e già introdotta nell'edizione critica pubblicata in CSEL 87. I 46 capitoli ancora considerati in analisi precedenti, sono ripartiti in 42 come segue: la Regula S. Augustini di prefazione al documento, prima fuori serie, diviene il capitolo I; i capitoli I-II, III-VI e XXX-XXXI unificati divengono rispettivamente i capitoli II, III e XXVII. Cf. anche: C. Leyser, Shoring fragments against ruin? Eugippius and the six-century of the florilegium, in: Eugippius und Severin. Der Autor, der Text und der Heilige, ed. W. Pohl - M. Diesenberger, Wien 2001, p. 68-70; J. Hofmann, Das Werk des Abtes 


\section{Le fonti della Regula Eugippii}

Una premessa si rende opportuna per apprezzare nel suo giusto valore la Regula di Eugippio, la quale, apparentemente come già si è accennato, non sembra un'opera originale, trattandosi di un centone di più scritti da diversi autori.

Nell'antichità cristiana, tale genere letterario, definibile "a compilazione" mediante trascrizione di brani da varie fonti inoltre non citate, era diffuso, apprezzato e, soprattutto, assai utilizzato. La sensibilità commerciale contemporanea lo accuserebbe di "plagio", ma in quei secoli privi di stampa, e in comunità preoccupate piuttosto dello scopo del bene collettivo che non della rivendicazione di singoli diritti o dei protagonismi privati, tutto ciò che poteva contribuire al giovamento pubblico, in tal caso morale, etico e spirituale, era salutato favorevolmente ${ }^{17}$.

Nella letteratura monastica, dove l'intento si prefiggeva la formazione alta e privilegiata di intere comunità di asceti, questo stile ebbe enorme fortuna: si trattava, infatti, di trasmettere da copista a copista il meglio di una tradizione, nel distillato di testi scelti e redatti a misura di una necessità precisa. Il tutto avveniva nella cornice carismatica di un'interpretazione dinamica della vita evangelica in sé. Qui si colloca perciò l'originalità di questo tipo di opere: quali frammenti di autori siano stati selezionati, perché quella scelta, come siano stati infine redatti insieme e con quale logica di sequenza e di composizione ${ }^{18}$.

Non fanno eccezione da ciò i brani biblici, di solito numerosissimi anche in quelle opere ritenute "originali" e aventi per autori insigni nomi

Eugippius. Zum literarischen Vermächtnis eines spätantiken Augustinus-Kenners an die frühmittelalterliche Kirche des Abendlandes, ZKG 109 (1998) p. 293-305; A. de Vogüé, Regula Eugippii, DIP VII 1575-1576; F. Villegas - A. de Vogüé, Praefatio, in: Eugippii Regula, CSEL 87, Vindobonae 1976, p. VII-XX.

17 Cf. Degórski - Mirri, Eugippio. La Regola, p. 18.

18 Cf. K. Obrycki, Wstęp, in: Eugipiusz. Żywot Ś'w. Seweryna. Reguła, ed. K. Obrycki, ŹM 13, Tyniec - Kraków 1996, p. 38-44. 
della letteratura patristica: non sarebbe difficile spesso poterle considerare "centoni" della Sacra Scrittura!19

Va inoltre ricordato che nell'ambito monastico era lontano il tardivo concetto giuridico di "regola". Per gli asceti, l'unica "regola" era la Bibbia, non lo scritto di un fondatore, la cui idea pure era assente secondo la moderna accezione. Dall'alveo della storia, la tradizione cristiana era percepita come la corrente vitale di un fiume, da cui scaturiva una esperienza redentiva continuata con sempre nuova freschezza da attingere. Fissare, quindi, di volta in volta quegli elementi che si adattavano alle esigenze presenti non significava codificarli nella fissità legislativa, ma offrirli ad un attivo patrimonio comune in evoluzione. Non interessava tanto la fonte o l'autore, quanto quello specifico contenuto da valorizzare e da non perdere, perché ritenuto un "dono" utile ed un aiuto tramandato per quel determinato momento del cammino cristiano. La Bibbia naturalmente ne restava il patrimonio fondamentale e il nucleo essenziale ${ }^{20}$.

Dalla Sacra Scrittura si traeva ogni indicazione e sapiente discernimento dell'unico punto principale: seguire e compiere la volontà di Dio. Il ruolo dell'abate o del superiore, nel primo percorso della vita cenobitica, si inscrive pertanto a questo servizio di garante dell'essere nella giustizia e nella verità di tale lettura e conseguentemente nella sequela della divina Parola. La "regola" è assente, perché sostituita dalla tradizione apostolica della prima comunità cristiana.

In una società religiosa, compaiono così piuttosto testi di spiritualità atti a riassumere e descrivere l'ermeneutica di quella esperienza, e ciò nelle citate forme di "detti", "colloqui" tra asceti in scambievoli consigli, epistole. Le "Regole" in quanto tali possono quasi interpretarsi come un'esegesi di tutto questo ricco patrimonio, un'antologia di una cono-

\footnotetext{
19 Cf. Degórski - Mirri, Eugippio. La Regola, p. 19.

20 Cf. Turbessi, Regole monastiche antiche, p. 11-32.
} 
scenza diretta di usi e pratiche cosolidate. Ognuna è una tappa di maggiore maturità convalidata dal vissuto ${ }^{21}$.

L'abate o superiore, perciò, venne ritenuto un padre spirituale di questa osservanza nei suoi confratelli, costituendosi più sull'autorità morale che giuridica, e formandoli più con 1'esempio che con la parola ${ }^{22}$. Egli, dunque, si presentava personalmente come "la prima Regola" o, ancora, l'incarnazione dell'unica vera "Regola": la Bibbia interpretata nell'insegnamento e nella vita dei santi maestri. La Parola di Dio e gli eccelsi modelli di santità hanno cominciato, allora, a significare la presenza di Cristo, fino a tradursi in ultimo nel motto: sub Regula uel abbate, sotto cioè l'autorità della Sacra Scrittura e la sua tradizione e quella di colui che con la condotta e la sapienza ne continuava a garantire l'osservanza. Obbedire alla Regula significò, dunque, obbedire all'abate, depositario della tradizione, organo della medesima e per ciò stesso regola vivente del monastero ${ }^{23}$.

In tale contesto di pensiero e di valori, tra le antiche regole religiose quella di Eugippio occupa allora una posizione di rilievo in quanto, con la sua datazione di origine tra il 530 e il 533, oltre che essere forse l'ultima precedente o addirittura contemporanea a quella di san Benedetto, che non cita mai, per la sua dimensione si presenta come una delle più lunghe pervenuteci, seconda soltanto alla Regula Magistri e alla Regula di san Basilio, di cui Eugippio usa la primissima versione, cioè il "Piccolo Asceticon"24.

21 Cf. Degórski - Mirri, Eugippio. La Regola, p. 20.

22 Cf. M. Bozzi - A. Grilli, Introduzione, in: Regola del Maestro, v. 1, ed. M. Bozzi - A. Grilli, Brescia 1995, p. 13-27.

23 Cf. A. de Vogüé, Sub Regula uel Abbate, in: Rule and Life. An Interdisciplinary Symposium, Massachusetts 1971, p. 21-63; S. Pricoco, Introduzione, in: La regola di S. Benedetto. Le regole dei Padri, ed. S. Pricoco, Milano 1995, p. IX-XVII.

24 Per ciò che riguarda la datazione delle Regula di Eugippio, cf. Degórski Mirri, Eugippio. La Regola, p. 20-21. Per un approccio del tutto inedito alla cronologia di Eugippio, si veda il recente articolo: A. Genovese, "Velut ex ingenti prato floribus asperso caelestibus". Gli Estratti dalle Opere di Sant'Agostino di Eugippio, "Urbaniana University Journal" 70/3 (2017) p. 177-215. 
Anche della Regula S. Augustini, unico testo riportato integralmente e collocato in apertura del florilegio, egli cita la versione più antica: quella dell'Ordo Monasterii, una sorta di brevissimo regolamento valido per comunità monastiche sia maschili che femminili, con l'abbinato testo conosciuto come Paeceptum ${ }^{25}$.

Oltre a questo scritto agostiniano, a 17 estratti da quello di san Basilio, 16 dell'anonima Regula Magistri, seguono 14 estratti dalle Conlationes Patrum e dal De institutis coenobiorum di san Giovanni Cassiano, e uno dalla Regula quattuor Patrum, uno dalla Regula di san Pacomio, uno dal sermone ai monaci di Novato e uno dalla lettera 125 di san Girolamo ${ }^{26}$. Escludendo il testo agostiniano, secondo un'analisi di A. de Vogüé, la fonte preferita da Eugippio fu la Regula Magistri: ne è infatti riprodotta circa un sesto, e occupa più della metà del codice Parisinus Latinus $12634^{27}$.

La ripartizione degli estratti per estensione di pagine (ciascuna implicante circa due fogli del Codex E) risulta come segue: Regula Magistri pp. 59, Regula S. Augustini pp. 22, testi di san Giovanni Cassiano pp. 20, Regula di san Basilio pp. 18, sermone di Novato pp. 12, Regula di san Pacomio pp. 2, lettera di san Girolamo p. 1 e Regula quattuor Patrum p. 1. Il Codex $E$ in totale è di circa 115 pagine, non calcolando l'estratto agostiniano. Nello schema di redazione dei frammenti, si può facilmente notare che la Regula Magistri si alterna con regolarità alle altre fonti del centone di Eugippio, costituendone come la spina dorsale. Nella prima ripartizione in 46 capitoli, anziché in 42, A. de Vogüé così presentava la suddivisione dei vari testi, sempre escludendo la Regula S. Augustini, data per preambolo "fuori serie": cap. 1: la Regula quattuor Patrum; cap.

25 Cf. Degórski - Mirri, Eugippio. La Regola, p. 21.

26 Cf. Turbessi, Regole monastiche antiche, p. 91-102 (introduzione legislazione pacomiana), 133-147 (introduzione legislazione monastica di san Basilio Magno), 269-280 (introduzione legislazione monastica di sant'Agostino), 317-327 (introduzione Regole dei Padri), 367-372 (introduzione alla Regula Magistri); Pricoco, Introduzione, p. XVIIIXXIV, cf. anche: 277, 282-283, 287-293, 312-317.

27 Cf. Degórski - Mirri, Eugippio. La Regola, p. 21. 
2: la Regula Magistri; capp. 3-5: san Basilio Magno; cap. 6: la Regula Magistri; capp. 7-19: san Basilio Magno; capp. 20-28: la Regula Magistri; cap. 29: san Pacomio; capp. 30-32: la Regula Magistri; cap. 33: Novato; capp. 34-42: san Giovanni Cassiano; capp. 43-44: la Regula Magistri; cap. 45: san Basilio; cap. 46: san Girolamo ${ }^{28}$.

La compilazione della Regula di Eugippio, pur essendo liberissima, non è priva di metodo: identità o affinità di argomento legano tra loro brani di autori distinti oppure di opere diverse, così come gli estratti da un'unica fonte possono costituire una serie di testi non spezzati, fornendo dei "blocchi" d'autore e opera, come accade per san Basilio e per san Giovanni Cassiano ${ }^{29}$.

È interessante osservare, per tipologia “ideologica", l'accostamento operato di diverse Regulae, poiché esse rappresentano la convergenza di due indirizzi spirituali differenti: l'uno improntato più sulla vita comune e l'amore fraterno (sant'Agostino, i “Quattro Padri”, san Basilio), l'altro privilegiante all'interno della comunità l'ordinamento gerarchico e la subordinazione al superiore (la Regula Magistri) ${ }^{30}$.

Un'altra tendenza viene poi decisamente fatta prevalere: quella che simpatizza per la vita cenobitica quasi a scapito addirittura della eremitica. A. de Vogüé osserva in proposito:

Citando in conclusione un brano in cui Basilio condanna ogni tipo d'isolamento, Eugippio intende chiaramente sbarrare la strada a quelli tra i suoi monaci

28 Cf. Degórski - Mirri, Eugippio. La Regola, p. 22.

29 Cf. A. de Vogüé, Nouveaux aperçus sur une règle monastique du VIe siècle, RAM 41 (1965) p. 19-29; per la ripartizione delle fonti nei 42 capitoli, cf. Obrycki, Wstep, p. $45-$ 47. In particolare, sant'Agostino occupa il cap. 1; la Regula quattuor Patrum 3 il cap. 2; la Regula di san Pacomio 159 il cap. 26; la Sententia di Novato il cap. 29; di san Giovanni Cassiano: la Conlatio 12, 2 il cap. 30; la Conlatio 12, 7 il cap. 31; il De institutis coenobiorum 4, 9 il cap. 32; il De institutis coenobiorum 4, 9 il cap. 32; il De institutis coenobiorum 4, 39-43 il cap. 33; il De institutis coenobiorum 2, 15-16 il cap. 34; il De institutis coenobiorum 3, 7 il cap. 35; il De institutis coenobiorum 4, 12 il cap. 36; il De institutis coenobiorum 4, 16 il cap. 37; il De institutis coenobiorum 4, 18 il cap. 38; la lettera 125 di san Girolamo il cap 42.

30 Cf. Degórski - Mirri, Eugippio. La Regola, p. 23. 
che sarebbero tentati di passare dal cenobitismo alla vita solitaria. La satira degli eremiti - presa da una lettera di Girolamo - che segue il lungo brano di Basilio, non lascia dubbi sulla prospettiva antieremitica di questa conclusione ${ }^{31}$.

In un altro saggio, dom A. de Vogüé aveva sottolineato già quanto l'autore del Codex $E$ fosse prevenuto verso la vita solitaria, sponsorizzando il pensiero di san Girolamo della Epistula 125, 9, in cui il Dalmata mette in guardia il giovane amico Rustico da un'esperienza anacoretica prematura, consiglio dettato forse dal ricordo della propria difficile esperienza in merito nel deserto di Calcide, all'indomani della propria conversione $^{32}$. Tuttavia, in quella lettera, san Girolamo non condannava affatto la vita eremitica in sé, bensì gli abusi deformanti la medesima.

Ebbene, Eugippio riproduce dell'epistola questa critica acerrima contro gli eremiti improvvisati, ma si astiene dal presentare la parte di testo geronimiano in cui sono definite le condizioni della legittima anacoresi, e cioè la lunga esperienza cenobitica e l'acquisizione di tutte le virtù, provate e fortificate nella vita comunitaria. A motivo di questa amputazione, nel testo di Eugippio, 'l'estratto di Girolamo appare come una condanna assoluta dell'eremitismo"33.

Stilisticamente parlando, infine, le analisi condotte sul Codex $E$ nel confronto con le sue fonti, rivelano modifiche significative di titoli ${ }^{34}$, interventi di perfezionamento su vocaboli ed espressioni, aggiunte di sette brevi frasi alla Regula di san Pacomio ${ }^{35}$ e un'eccedenza di citazioni bibliche rispetto alla Regula Magistri ${ }^{36}$.

31 A. de Vogüé, L'influenza di Basilio sul monachesimo occidentale, in: Basilio tra Oriente e Occidente. Convegno Internazionale "Basilio il Grande e il Monachesimo Orientale”, Cappadocia, 5-7 ottobre 1999. Comunità di Bose, ed. S. Brock - M. Cortesi - A. de Vogüé - J.-R. Pouchet, Magnano 2001, p. 219.

32 Cf. L. Mirri, La dolcezza nella lotta, Bose 1996, p. 15-22.

33 de Vogüé, Nouveau aperçus, p. 30.

34 Cf. per esempio capp. XXVIII, XXIX e XLVI della Regula di Eugippio.

35 Cf. Eugippius, Regula XXVI 40-46.

36 Cf. Eugippius, Regula XXII 6 - XXIV 10-11; de Vogüé, De Cassien au Maître: le titre du chapitre de l'humilté, SMon 24 (1982) p. 247-261. 
L'opera di Eugippio respira di passi tratti dalla Sacra Scrittura: alcuni di essi erano già incorporati nelle fonti che egli utilizza, moltissimi altri sono sue aggiunte convalidanti un principio spirituale insegnato, oppure incoraggianti con l'autorità della Parola di Dio la "fatica" ascetica e il cammino per l'evangelica "via stretta".

In sintesi, il testo biblico in assoluto più citato è il Salterio. Se si considera che anche la tematica più presa in esame nella Regula di Eugippio è la preghiera, che nello scopo della vita del monaco dovrebbe essere "incessante", ciò non può destare meraviglia, ma rappresentare una coerente sintonia logica. Recitati a memoria giorno e notte, i salmi dovevano essere, anche per Eugippio e i suoi monaci, non solo la chiave esegetica dell'intera Bibbia, bensì pure l'ermeneutica della loro vita monastica. Nella sequela di Cristo, Parola del Dio vivente, l'uomo di Dio si sentiva chiamato a divenire preghiera vivente di quella medesima Parola da cui riceveva grazia e verità per la sua catarsi nell'amore divino.

Tra i libri dell'Antico Testamento, più citati sono, quindi, i Proverbi e il Siracide, fonti della sapienza del monaco ${ }^{37}$; dal Nuovo Testamento, il Vangelo di san Matteo con il suo annunzio prorompente e vigoroso del Regno dei cieli e due lettere di san Paolo: quella ai Romani e la prima ai Corinti, l'una per il tema Legge e Spirito, l'altra per il primato che annuncia, quello della carità. Esso è infatti assai caro ad Eugippio, che lo presenta in apertura e in conclusione della sua Regula, offrendo così chiaramente il cuore del proprio pensiero: la sequela di Cristo è possibile attraverso la regola d'oro del Vangelo, che è la realizzazione del comandamento dell'amore di Dio e del prossimo.

37 Cf. B. Degórski, I Libri sapienziali nella letteratura monastica delle origini (secc. IV-V), in: Letture cristiane dei Libri Sapienziali, SEA 37, Roma 1992, p. 65-86; B. Degórski, L'elenco delle citazioni dei Libri sapienziali nella letteratura monastica del IV e V secolo, RT 40 (1993) p. 119-154. In questa letteratura ascetica maturata in ambienti maschili, è assente il Cantico dei Cantici, considerato troppo sensuale. 


\section{Le tematiche teologiche della Regula Eugippii}

Per una conoscenza appropriata del contenuto d'insieme della Regula di Eugippio, essendo essa stata stilata per la comunità erede della spiritualità ascetica di san Severino, è utile inserirla nell'orizzonte dell'esperienza monastica dell'istituzione severiana medesima. Dal qualificato studio della Vita S. Severini condotto da Ph. Régerat ${ }^{38}$, si apprende che quel monachesimo sviluppatosi nel Norico aveva la caratteristica di un'organizzazione a forma di colonie semieremitiche di tipo orientale.

Nella biografia, Eugippio mai chiama san Severino con il titolo di abbas, bensì di doctor, esprimendo in ciò la stima per il maestro di perfezione e la relazione di un padre spirituale verso i propri figli. L'idea cenobitica resta esplicitamente sfumata ed Eugippio coglie assai bene la pedagogia e l'ideale di Severino: la comunità non può essere formata dall'esterno per volontà di un fondatore più o meno dotato di carisma e di personalità, mettendo insieme singoli individui che tali restano seppur tra loro aggregati.

I termini congregatio e societas, spesso accompagnati dall'aggettivo sancta, che Eugippio impiega nella Vita S. Severini, e la sottolineatura degli sforzi permanenti del Santo del Norico per creare, promuovere, nutrire e preservare l'unità dei propri discepoli con l'amore fraterno, rimanda direttamente al modello apostolico della prima Chiesa gerosolimitana (cf. At 4, 32).

Siamo così posti dinanzi alle tre grandi coordinate teologiche della Regula di Eugippio: il modello ecclesiale della prima comunità cristiana come prototipo della comunità monastica, l' "abate" inteso come doctor-maestro e il fondamento della carità, che è il vincolo di perfezione. Perfetta coesione di elementi di trinitaria, cristologia, pneumatologia

$38 \mathrm{Cf}$. Ph. Régerat, Introduction, in Eugippe. Vie de Saint Séverin, ed. Ph. Régerat, SCh 374, Paris 1991, p. 122-135. Lo studio è di importanza capitale per la Vita S. Severini, ma getta anche tanta luce e fornisce importanti indizi per conoscere sia lo stesso Eugippio che specialmente il contesto ecclesiale in cui egli viveva. 
ed ecclesiologia sono alla base di un pensiero di spiritualità monastica che vede promossa una nuova antropologia teologica in cui la persona, come vedremo, risulta ampiamente riscattata e valorizzata sulla positiva fiducia in lei riposta.

La carità è il principio costituente al suo interno la sancta societas e, in essa, si fonda pure il servizio paterno del superiore prepostovi. La dimensione di vita cenobitica del gruppo si forma, dunque, e si alimenta interiormente, tessuta spontaneamente nei rispettivi rapporti di reciprocità fraterna dalla plasmazione in tutti e in ciascuno dell'amore divino.

Con stile giovanneo "ad anello", Eugippio dà avvio alla sua Regula con il testo di Mt 22,37-40 sul comandamento più grande: 1'amare Dio con tutte le proprie forze ed il prossimo come se stessi. Termina poi nel penultimo capitolo con un elogio dell'esercizio della carità praticato nella vita comunitaria ${ }^{39}$. È palese, perciò, che dall'esordio alla fine del suo centone, la logica prevalente nella cernita delle fonti dalle quali attinge, ed il conseguente leitmotiv dell'intera opera, sia la carità, "vincolo di perfezione", virtù teologale per eccellenza e habitus interiore da cui deriva ogni prassi genuinamente cristiana.

Qui sta la novitas evangelica della salda unità della Chiesa primitiva, la quale compatta seppe affrontare persecuzioni e difficoltà, e superare pregiudizi e diversità tra i propri membri, di culture e provenienze giudaiche e pagane insieme. La comunità monastica non si presentava differente per problematica. Tempi travagliati per l'impatto con popoli nuovi e un cristianesimo assunto a religione dell'Impero rendevano eroica la testimonianza ascetica: la lotta ai vizi, ai compromessi degli agi apportati da libertà e potere e a tanti sviamenti morali e dottrinali dominanti anche all'interno dell'istituzione ecclesiale esigevano fortezza e rettitudine inflessibili e discernimento illuminato.

Quanto a provenienza, pure i monaci di una medesima comunità potevano trovarsi a convivere tra confratelli di assai diversa estrazione

39 Cf. Eugippius, Regula I 1 e cap. XLI. 
sociale e culturale, oltre che di disparata esperienza umana e cristiana, inclusa quella dei grandi convertiti. Eugippio seleziona brani in cui ciò si percepisce ${ }^{40}$. Il monastero allora guarda alla Chiesa apostolica e su quell'esempio si preordina come schola Christi ${ }^{41}$. A riguardo, chiarificante è la considerazione con la quale G. Penco conclude un proprio saggio sull'argomento:

La "Regola dei monasteri" come norma di vita spirituale per ogni epoca e ambiente è la risultante di tutta l'esperienza ascetica che non rimane necessariamente legata ad una regola, per quanto importante essa possa essere, ma che deve riflettere l'insieme di questo insegnamento nella coralità delle sue voci. E ciò rientra in una ineliminabile e profonda considerazione pedagogica della vita religiosa, di quella "schola religionis" che deve educare specialmente i giovani monaci per portarli alla pienezza della loro formazione spirituale. Solo in tal modo si possono evitare i pericoli dell'autodidattismo e, per conseguenza, del dilettantismo, non meno pericolosi nella vita spirituale di quanto non lo siano negli altri settori dell'umana esistenza ${ }^{42}$.

Abbinato a tale idea della comunità monastica, va aggiunto il concetto di monastero inteso come Ecclesia Christi, ancora sulla tipologia della Chiesa delle origini e sulla scia nostalgica di un ideale puro di sancta societas nella koinonia di molti fratelli. In questo contesto prende fisionomia il "superiorato".

Ai profeti guide dell' Israele veterotestamentario e agli Apostoli guide del nuovo Popolo di Dio, succedono anche per Eugippio i doctores, guide dei monasteri, ma con una peculiarità. L'abate di Lucullano inverte l'ordine profeti/apostoli in apostoli/profeti, ai quali fa seguire la categoria dei dottori. Egli, infatti, fa riferimento a "tre gradi di magistero" costituiti dal

40 Cf. in particolare: Eugippius, Regula I 35-46; G. Penco, La composizione sociale delle comunità monastiche nei primi secoli, SMon 4 (1962) p. 257-281.

41 Cf. Eugippius, Regula XXVII 27.

42 G. Penco, Sul concetto del monastero come "schola”, CCist 32 (1970) p. 333. 
Signore per la sua Chiesa in nome della Trinità ${ }^{43}$. Se è evidente che gli apostoli corrispondono ai vescovi nella Chiesa e i dottori agli abati nei monasteri, i profeti posti in medio intendono probabilmente richiamare agli uomini di Dio dell'una e dell'altra “classe" la dimensione carismatica e l'impegno santificante per sé e per il prossimo, cui nessuno può esimersi.

Umiltà, castità, penitenza, preghiera, giustizia, obbedienza, silenzio, carità nell'abate devono eccellere in quanto più degli altri monaci è tenuto a dare l'immagine dell' "uomo celeste". Egli è costituito come perno e riferimento di una sorta di mistica comunitaria che scaturisce dall'unione di ciascuno con Dio. Infine, essendo pure colui che fa le veci di Cristo, l'abate è magister delle virtù nell'esemplarità concreta, praticandole "per" e "dinanzi" ai suoi monaci, quasi spianando la "via stretta" della radicale sequela del Signore, a modo di pastore in testa al proprio gregge. Eugippio, in un testo scelto, lo appella splendidamente: "artigiano della santa arte", intendendo per quest'ultima la vocazione monastica ${ }^{44}$.

Il tutto corrisponde all'idea severiana dell'abate come "padre carismatico" posto a capo di un'autentica comunione di fratelli, prima ancora che di una "comunità". Egli è tenuto a occuparsi personalmente della loro dimensione spirituale, sia personale che collettiva, cercando di trasmettere e inculcare l'eredità dei santi Padri, tra i quali erano stimati con preferenza quelli orientali.

La tradizione ascetica dell'Oriente cristiano entrò nelle Regole diffuse in Occidente e, attraverso l'Ordo Monasterii e il Praeceptum agosti-

\footnotetext{
43 Cf. Eugippius, Regula XXVII 26.

44 Cf. Eugippius, Regula XXV 21; per l'abate come "padre": I 3 e XXIX 26; per l'abate che fa le veci di Cristo: XXV 2; per l'abate come "pastore": XXV 7 e 10; per l'abate come "maestro": XXV 11, 17, 20 e cap. XXVI; ancora sul "superiore": I 140-148. Cf. anche P. Tamburrino, La Regula Magistri e l'origine del potere abbaziale, CCist 28 (1966) p. $160-173$.
} 
niani ${ }^{45}$, anche nel centone di Eugippio, come testimonia l'orario liturgico di ripartizione della preghiera e della giornata in genere $^{46}$.

Quanto alle virtù ascetiche, l'esperienza inagurata da san Severino privilegiava l'umiltà di cuore, la giustizia, il fervore interiore animato dalla compunzione e dalla preghiera continua, e la castità. Sono punti di rilievo anche nella compilazione dell'abate di Lucullano. Per 1'ultimo citato, egli adotta completamente, nei capitoli XXX e XXXI della sua Regula, le Conlationes 12, 2 e 12, 7 di san Giovanni Cassiano, mentre l'argomento "giustizia" è fortemente presente nel testo agostiniano del Praeceptum nn. 53-90. Questo non solo è copiato integralmente, ma è ritenuto molto importante per l'equilibrio e la moderazione cui educa.

Per esempio, trattando del digiuno, tematica sulla quale la Regula non si soffermerà ulteriormente, lo consiglia tra i mezzi ascetici classici per il dominio di sé, ma solo "per quanto la salute lo permetta" 47 . Altrettanto può dirsi per la distribuzione di cibo e di vestiario, da darsi "a ciascuno secondo le necessità"48. Equità, ancora, è usare trattamento differenziato per chi viene da abitudini agiate, dovute alle ricchezze delle quali era favorito nel mondo, e ovviamente per chi è afflitto da malattia: il principio è che a nessuno deve essere posto sulle spalle un peso superiore alle proprie capacità. Infatti, il mezzo non va confuso con il fine e l'ascesi in sé è soltanto strumento di perfezione.

45 Cf. B. Degórski, Il primissimo monachesimo nell'Africa Latina, VoxP 53-54 (2009) p. 591-598; B. Degórski, św. Augustyn z Hippony - krzewiciel monastycyzmu w Afryce Łacińskiej, in: W pedagogicznej służbie narodów, ed. M. Włosiński, Włocławek 2009, p. 123-133; B. Degórski, De Sancto Aurelio Augustino Episcopo Hipponensi veritatis Christianae defensore eximio, in: Nauka o wymiarach prawdy. Studio sulle valenze della verità. Rozmery pravdy. Księga Pamiątkowa z okazji 70. Rocznicy Urodzin ks. Profesora Mariana Włosińskiego, ed. S. Kunikowski, Włocławek 2015, p. 103-115.

46 Cf. Eugippius, Regula I 2; X 24; A. de Vogüé, L’influence du monachisme des Kellia en Occident, in: The Spirituality of Ancient Monasticism, p. 164-168; A. de Vogüé, L'horaire de l'Ordo Monasterii. Ses rapports avec le monachism égyptien, in: Homo Spiritalis, Würzburg 1987, p. 240-258.

47 Eugippius, Regula I 53.

48 Eugippius, Regula I 33. 
Eugippio pare preoccupato anche di esorcizzare nella comunità il serpeggiare di malumori, gelosie e mormorazioni ${ }^{49}$ e non lesina pertanto frammenti che facciano riflettere i monaci sulla prospettiva capovolta in cui tenere in considerazione le cose rispetto alla mentalità secolare: attenzioni particolari e attenuazioni di asperità di vita non sono un vantaggio e un onore nella vita monastica, bensì quai un'umiliazione, perché rappresentano una concessione a debolezza e fragilità. Fortunato è, dunque, chi non ha bisogno di dispense, benefici ed esenzioni da alcune pratiche ascetiche ${ }^{50}$.

Lo stesso va detto per la correzione fraterna, di carattere "medicinale", anche preventivo se necessario, ma non punitivo ${ }^{51}$. Le sanzioni, d'altronde, nell'intera Regula, concernono quasi esclusivamente il problema di un colpevole ritardo alla preghiera, vero nucleo della vita monastica personale ecomunitaria ${ }^{52}$. Meno egli si occupa di altre mancanze, ritenute malattie da guarire quanto prima ${ }^{53}$. Qui entra in gioco la formazione della sincerità e umiltà di cuore, le sole virtù in grado di combattere e vincere i vizi con l'aiuto di Dio e pure dei confratelli, tramite aperta confessione e pronta obbedienza al superiore ${ }^{54}$.

Per l'intero capitolo XXIX, dedicato tutto al problema di umiltà e obbedienza contro orgoglio e superbia, Eugippio si avvale della omonima sentenza di Novato, esplicita ed esauriente a riguardo ${ }^{55}$. Ma il testo certamente "gioiello" della Regula, e probabilmente per tale suo valore redatto da Eugippio al centro del centone, è il cap. XXVIII interamente tratto dalla Regula Magistri e avente per tema la grazia dell'umiltà e del progresso verso Dio.

49 Cf. Eugippius, Regula XXIX 76-81.

50 Cf. Eugippius, Regula I, 59-68.

51 Cf. Eugippius, Regula I 85-90.

52 Cf. Eugippius, Regula XXI 34-35.

53 Cf. Eugippius, Regula XXIX 52-59.

54 Cf. Eugippius, Regula XVIII e XXXII.

55 Cf. A. de Vogüé, Novati Sententia de Humilitate et Obedientia, DIP VI 441442; A. Solignac, Novat le catholique, DSp XI 477-478; T. Kardong, Justitia in the Rule of Benedict, SMon 24 (1982) p. 43-73. 
L'immagine della scala del sogno di Giacobbe (cf. Gn 28,12) è ivi presentata nell'abile ermeneutica della frase evangelica: "Chiunque si esalta sarà umiliato, e chi si umilia sarà esaltato" (Lc 14,11), non senza lo sfondo esegetico sotteso della grande testimonianza del Battista: "Nessuno può prendersi qualcosa se non gli è dato dal cielo [...]. Egli deve crescere e io invece diminuire" (Gv 3,27.30). L'umiltà come grazia, alla quale l'uomo può corrispondere apportando le condizioni del primato di Cristo in sé, e la perfezione come come conseguimento di quel medesimo primato in ogni intento, pensiero e fine d'amore del monaco, si connettono dalla metà del centone al suo punto di partenza, con Mt 22,37-40, e al suo punto di epilogo, con Col 3,14 da mettere in pratica.

Non è perciò casuale la collocazione di questi tre testi nella compilazione dell'abate di Lucullano, il quale ha palesemente elaborato per temi favoriti una cernita mirata di pagine da opere di altissima spiritualità e di efficace insegnamento sulla sequenza ermeneutica di un messaggio evangelico portante: la carità.

Accostando infatti i contenuti dei capitoli XXVIII e XIX della Regula, ci si accorge come la dinamica del pensiero presentato intenda sviluppare una formazione nella quale l'umiltà induce all'obbedienza e questa ha radici nella carità vera, che ottiene nella prassi comunitaria povertà e sensibilità interiore per il fratello. Questo affinamento in carità è "il vincolo di perfezione", in quanto è la perfetta imitazione di Cristo e l'incarnazione dell'amore teandrico che scorre nel corpo stesso di Cristo: la Chiesa-comunità. Umiltà e carità sono il culmine e lo stupore della cristologia paolina, che proprio sull'esortazione ad avere interiormente "gli stessi sentimenti che furono in Cristo Gesù", il quale per amore dell'uomo non "considerò tesoro geloso" la propria divinità, ma ne rese partecipe la creatura spogliando se stesso e facendosi obbediente al Padre fino alla morte più ignominiosa (cf. Fil 2,5-8), riportò in sé gli uomini all'unità con Dio e tra di loro. Fare la volontà di Dio e sacrificare la propria, in una mistica crocifissione di quest'ultima nel- 
la carità, è la condizione sine qua non per un'armonica vita comunitaria edificata sull'esercizio delle virtù ${ }^{56}$.

Seguendo, infine, a conclusione ormai del testo, l'ecclesiologia paolina del corpo mistico di Cristo, in cui tutti si è membra l'uno dell'altro (cf. Rm 12,5), e utilizzando e rielaborando un frammento di san Basilio, Eugippio lascia il suo intenso messaggio spirituale alla comunità severiana: "Ci dobbiamo adattare con armonia, ed essere ben uniti nello Spirito Santo come nell'organismo di un solo corpo" ${ }^{57}$. Egli lancia quindi il suo inno alla carità con quel brano geronimiano che trasforma in un'esaltazione della vita cenobitica per il motivo della superiorità, in essa, della carità praticata e della carità, si può dire "moltiplicata", tra i fratelli che, come una milizia compatta, con essa e per essa danno l'assalto al Regno dei cieli (cf. Mt 11,12) ${ }^{58}$.

\section{Messaggio e attualità della Regula Eugippii}

I contenuti privilegiati da Egippio sono subito tutti presentati nella cosiddetta Regula di Agostino, sua fonte prediletta per gettare le basi di

56 Cf. Eugippius, Regula XXXIII.

57 Eugippius, Regula XLI 18.

58 Nel centone, in più parti, è usato il linguaggio militare, classico della letteratura monastica nella quale l'asceta, oltre che atleta di Cristo, è perantonomasia chiamato il miles Christi. Cf. Eugippius, Regula XLII 90-95 per il tema della superiorità della carità nella vita cenobitica. Per un commento alla Regula di Eugippio cf.: M. Krausgruber, Die Regel des Eugippius. Die Klosterordnung des Verfassers der Vita Sancti Severini im Lichte ihrer Quellen. Text, Übersetzung und Kommentar, Innsbruck 1996, p. 215-253; A. de Vogüé, Les conseils évangéliques chez le maître et saint Benoit, in: Los consejos evangélicos en la tradicin monástica, Abadia de Silos 1975, p. 13-27; A. de Vogüé, Cassien, le Maître et Benoît, in: Commandements du Seigneur et libération évangélique, Roma 1977, p. 223-235; D. Sanchis, Pauvreté monastique et Charité fraternelle chez saint Augustin, "Augustiniana" 8 (1958) p. 5-21: questi studi mettono in luce le fonti utilizzate da Eugippio (l'Ordo Monasterii, la Regula Magistri e san Giovanni Cassiano), gli aspetti di teologia monastica e teologia spirituale esposti. Sui contenuti della Regula Magistri, cf. anche: I. Gobry, Storia del monachesimo, v. 1, Roma 1991, p. 680-694; A.A. Häussling, Das Commemoratorium des Eugippius und die Regula Magistri und Regula Benedicti, in: Regulae Benedicti Studia, ed. B. Jaspert - E. Manning, Annuarium Internationale 5, Hildesheim 1977, p. 33-42. 
una profonda spiritualità monastica sui suoi elementi principali: preghiera, lavoro, povertà, obbedienza, silenzio, direttive per il pasto e i rapporti con il mondo esterno. È indicato anche l'obiettivo di questa formazione: l'unità cristiana delle anime e dei cuori. Viene incoraggiata una tipologia "familiare" quasi della comunità, in cui i luoghi adibiti per i servizi comuni come l'oratorio o il refettorio sono al centro dell'attenzione tanto quanto i rapporti fraterni.

Tutto questo comporta che siano sufficienti norme generiche, prive di dettagli precisi anche per ciò che concerne un certo ordinamento pratico dei compiti, dei ruoli o di altro ancora. La responsabilità di ciascuno diviene corresponsabilità reciproca affidata alla crescita e alla maturità della cosciena del singolo monaco, che soltanto così si affina sia umanamente, sia spiritualmente.

Sensibilità interiore ed educazione costituiscono un binomio imprescindibile ed essenziale nella chiamata alla santità, perché indici dell'irrorazione feconda della grazia che viene dall' "alto" e della sinergia attiva della creatura che si lascia plasmare dalla carità.

Il come trattare gli attrezzi da lavoro può sembrare un particolare irrilevante nella Regula ${ }^{59}$, che qui utilizza un brevissimo brano della Regula Magistri, ma in realtà non è che una sfumatura di quel riguardo squisito e discreto, umile e costruttivo che tesse fibre robuste di solidarietà, in comunità, assai più intime e intense. Per esempio, un dovere fraterno è, in questo spessore di relazioni, quella vigilanza l'un l'altro che può prevenire peccati e insidie del male nel confratello ${ }^{60}$ : è la carità per la quale, come un angelo custode, il fratello protegge la santità del fratello.

L'intento della Regula, e il ruolo che Eugippio le attribuisce per i suoi monaci, è quello di essere uno strumento della via al cielo. Ne deriva la sua elasticità e adattabilità alle situazioni singole o comunitarie che possano presentarsi nella dinamica di un cammino ascetico condiviso.

\footnotetext{
59 Cf. Eugippius, Regula III 3-16.

60 Cf. Eugippius, Regula I 83-96.
} 
Volendo soprattutto formare una coscienza, ancor prima di una volontà, essa attenua gli aspetti pratici e disciplinari, relegati i primi a rango di elementi secondari, gli altri di estremo ricorso in un contesto in cui si presuppone applicato il comandamento dell'amore a Dio e al prossimo.

Non va pertanto trascurato il passo agostiniano a conclusione del Praeceptum in cui si esortano i monaci a leggere una volta alla settimana quel "libello", affinché possano mirarvisi "come in uno specchio"61. È su questa premessa che Eugippio procede alla selezione sintetica e qualitativa di quanto ha globalmente annunciato con il testo di sant'Agostino per la crescita spirituale dei suoi confratelli. Con frammenti da varie opere egli sviluppa idee che ritiene importanti fissare in loro a comune edificazione. Probabilmente è tale concezione di Regula come speculum della propria santità in itinere che può aver suggerito all'abate di Lucullano di inserire nel suo centone un ampio passo della Regula Magistri di sapore prettamente escatologico ${ }^{62}$.

Un po' oltre la metà dell'opera, si apre infatti, quasi ad incoraggiamento del cammino verso l'ardua meta del paradiso, uno scorcio di visione della realtà del Regno dei cieli, dove il trionfo della carità fuga il timore e l'armonia della nuova creazione abbraccia quei puri di cuore ammessi alla comunione dei santi, alle immortali ricchezze e all'unione perpetua con Dio. La Regula, dunque, si propone anche come "specchio" della meta: specchio rifrangente della bellezza e della gloria che sarà, divenendo nella teologia monastica l'organo che fissa non solo la memoria del passato sulle orme dei Padri e del presente nella propria storia di salvezza, ma anche la memoria del futuro, della quale l'asceta deve costituire la testimonianza vivente e da cui trae tutto il senso ed il significato della sua scelta e della sua esistenza.

Riassumendo i temi esposti dalla Regula di Eugippio, essi si dipanano come segue, secondo la categorizzazione datavi da M. Krausgruber:

\footnotetext{
61 Eugippius, Regula I 152.

62 Cf. Eugippius, Regula XXVIII 87-114.
} 
istruzioni fondamentali; istruzioni pratico-organizzative (suddivisione del giorno, servizio divino [con indicazioni sulla ripartizione dei salmi, comportamento e dispense varie], governo della comunità, ruoli, lavoro, refezione, vestiti, malattie, comportamento da tenersi con il mondo esterno); conseguimento della perfezione (elenco dei vizi (superbia, ostinazione), combattimento contro gli stessi, trattazione delle virtù [umiltà, obbedienza, castità]; scuola del servizio di Dio (funzione del monastero sulla via del cielo [la via della perfezione, l'ascesi, il comportamento con i beni materiali], la guida spirituale, la comunità [predilezione della vita comunitaria, comportamento da tenersi davanti alle mancanze dei fratelli]; aspetti disciplinari ${ }^{63}$.

Ancora dalla ricerca di M. Krausgruber, è utile prendere in esame le percentuali di quanto ciascun tema si presenta trattato nella Regula di Eugippio. Ciò concede di procedere più chiaramente ad una lettura del messaggio ultimo dell'abate di Lucullano, la struttura del cui centone dimostra, infine, originalità di pensiero, come si potrà cogliere da un confronto sinottico con la Regula Magistri e la Regula di san Benedetto, riguardo alla ripartizione degli stessi contenuti di base ${ }^{64}$.

Negli schemi compilati dallo studioso austriaco le proporzioni rendono il peso di un argomento nelle opere prese in esame. Come premessa va intanto evidenziato che nella Regula di Eugippio i temi fondamentali occupano 48 versetti, quelli spirituali 650, quelli pratico-organizzativi 249 e quelli disciplinari 160. Analisi parallela è stata condotta sulle "Regole" del Maestro e di san Benedetto. Traducendo, quindi, in percentuale la presenza delle singole tematiche in ogni Regula, emerge quanto segue: 1) Temi fondamentali: a. la Regula di Eugippio circa il 4\%; b. la Regula Magistri circa il 4\%; c. la Regula S. Benedicti circa il 2\%. 2) Temi spirituali: a. la Regula di Eugippio oltre il 58\%; b. la Regula Magistri circa il 28\%; c. la Regula S. Benedicti circa il 37\%. 3) Temi pratico-organiz-

\footnotetext{
63 Cf. M. Krausgruber, Die Regel des Eugippius, p. 255-277 (analisi della Regula).

64 Cf. Krausgruber, Die Regel des Eugippius, p. 278-282, 305-309.
} 
zativi: a. la Regula di Eugippio meno del 24\%; b. la Regula Magistri circa il 64\%; c. la Regula S. Benedicti circa il 55\%. 4) Temi disciplinari: a. la Regula Eugippio circa il 14\%; b. la Regula Magistri circa il 4\%; c. la Regula S. Benedicti circa il 6\%.

Rispetto alla sua fonte principale per estensione, la Regula Magistri, e al testo coevo della Regula S. Benedicti, la Regula di Eugippio si impone nettamente per predilezione del soggetto spirituale, mentre risulta decisamente inferiore per la trattazione di quello pratico-organizzativo ${ }^{65}$.

Valutando, in ultimo, soltanto all'interno della Regula di Eugippio la ripartizione in percentuale degli argomenti trattati nei due sottogruppi principalmente considerati si ha: 1) Temi spirituali: vizi $8 \%$; virtù $33 \%$; Ufficio divino $3 \%$; via di perfezione $16 \%$; ascesi $1 \%$; uso beni materiali $4 \%$; guida spirituale $4 \%$; comunità $25 \%$. 2) Temi pratici: suddivisione del giorno $11 \%$; servizio divino $28 \%$; governo della comunità $7 \%$; ruoli e funzioni $11 \%$; lavoro $19 \%$; refezione $11 \%$; vestiario $7 \%$; malattia $3 \%$; comportamento con l'esterno $3 \%$.

I temi spirituali offrono un chiaro messaggio del pensiero dell'abate di Lucullano il quale, attribuendo ben il 33\% di spazio tra di essi alle virtù, contro soltanto 1 ' $8 \%$ conceso all'argomento dei vizi, svela la sua impostazione prettamente positiva nella considerazione della formazione interiore della persona. La proposta di ideali alti da conseguire e la promozione delle qualità migliori dell'individuo sono, nel suo pensiero cristiano e monastico, certamente più avvincenti e stimolanti di un'ascetica o morale "negativa", tese più a "paralizzare" il soggetto che ad attivarlo nel meglio di sé con fiducia nelle proprie potenzialità. Anche in questo messaggio, pare comunque di cogliere il suo tesaurizzare il principio evangelico della carità, liberatorio la creatura con l'esortazione: "Tutto quanto volete che gli uomini facciano a voi, anche voi fatelo a loro: questa infatti è la Legge e i Profeti” (Mt 7,12).

65 Cf. anche Pricoco, Introduzione, p. XXXVII-XLIII, per un confronto fra la Regula di san Benedetto e quella del Maestro. 
Per ciò che riguarda il dato che all'ascesi egli abbia concesso, all'interno di una regola monastica, solo 1' $1 \%$ di spazio percentuale sull'intero corpus dell'opera, in assoluto la più bassa per ogni tema da lui toccato sia tra quelli spirituali, sia tra quelli pratici, non può che far riflettere. Si è senz'altro posti dinanzi alla peculiarità di maggior spicco dello scritto, in considerazione del suo genere e, pertanto, più indicativa della personalità spirituale di Eugippio, la quale si configura di tipo mistico-intuitiva volta verso 1'“alto"e non ascetico-analitica nell'ispezione di sé.

Il primato della carità, l'umiltà come grazia, una formazione volta più alla prassi fraterna e comunitaria che alla preoccupazione per la propria satificazione sono elementi in se stessi indici di una "mistica dell'ascesi" assai matura sulla conversione al Vangelo di Cristo. Il rapporto fraterno e comunitario anche si evidenzia come luminoso riverbero dell'autentica unione con Dio vissuta in semplicità come dono e gratuità, piuttosto che come meta ambita da acquisire con le proprie forze.

In tale ottica, il mezzo ascetico viene naturalmente superato così come la legge è vinta in generosità dall'amore. L'umiltà che fa scendere la scala di Giacobbe, e non salire, è eloquente su questo insegnamento spirituale trasmesso da Eugippio. Volersi "far santo" da sé con l'imposizione di pratiche ascetiche straordinarie o stravaganti, che nella sua Regula proibisce drasticamente quanto le forme eccentriche di vita monastica $^{66}$, è inganno diabolico contrario alla carità e peccato di presunzione. L'umiltà che fa diminuire l'ego e crescere Cristo conduce al perfetto svuotamento di sé nella kenosis radicale. Allora il monaco sarà all'apice della scala di Giacobbe, come Cristo innalzato sulla croce: nella luce del Padre e nell'ora della gloria, a cui la morte introdurrà.

La vita cenobitica ha valore nel pensiero di Eugippio in quanto è palestra del lasciarsi lavorare dalla grazia intimamente. Silenzio, obbedienza e confratelli sono i mezzi ascetici privilegiati e corroboranti tale cammino di perfezione evangelica. Per questo, ancora, ogni buona ragione

${ }^{66}$ Cf. Eugippius, Regula V e XXIII; XVIII 18-48; XXVII 6-18. 
indirizzata allo stesso scopo può costituire un'eccezione alla regola stessa, come per esempio il silenzio durante il lavoro o il riposo notturno ${ }^{67}$.

Persino la castità viene presentata con testi delicati che ne fanno percepire il valore positivo per il bene e l'equilibrio di se stessi. Non vi sono accenti di disprezzo della donna, ma di sincerità interiore a cui educarsi per un autentico cuore puro. I contatti con la donna non sono in sé proibiti da strette clausure, ma concessi sotto vigilanza interiore e con onesta prudenza. Inoltre è stupendo il brano che, su tale questione, s'incontra e che, sulla scia del Salmo 132, 1, celebra con il massimo encomio il valore della vita fraterna nel cenobio: "custodite reciprocamente la vostra pudicizia. Infatti, Dio che abita in voi, anche in questo modo vi proteggerà, ossia per mezzo di voi stessi" ${ }^{\prime \prime}$. Sembra di leggere l'antidoto delle conseguenze del primo peccato: "Il Signore disse a Caino: Dov'è Abele, e tuo fratello? Egli rispose: Non lo so. Sono forse il guardiano di mio fratello?" (Gn 4,9). Nell'economia della Redenzione, nuovamente il fratello è "il guardiano" di suo fratello, nel nome - nella presenza, cioè, secondo l'accezione biblica! - del Signore.

Ecco allora motivato da una salda teologia il secondo dato che emerge in percentuale nella Regula: la comunità. Con il 25\% di spazio concessole nel testo è da catalogarsi per importanza al terzo posto, dopo le virtù, mentre, tra i temi pratici, seguono le norme per il servizio divino che si evidenziano con il 28\%. Seguono, infine, ancora tra i temi pratici, il lavoro con il 19\% e, tra gli spirituali, quanto concerne la via di perfezione, con il 16\%. Tutto il resto appare come un corollario all'uno e all'altro settore. Si può comunque osservare, tra i temi pratici, che quello del servizio divino è il più attinente all'ordinamento spirituale e quello del lavoro a ciò che concerne la comunità ${ }^{69}$.

\footnotetext{
7 Cf. Eugippius, Regula I, 26; XXII.

8 Eugippius, Regula I, 83.

69 Cf. Degórski - Mirri, Eugippio. La Regola, p. 41.
} 
È evidente, dunque, la conferma di una linea precisa del messaggio di Eugippio nel suo centone, il cui valore è quello di un'opera monastica non parca di originalità. Più vicina ad un timbro spirituale dell'Oriente cristiano, l'insegnamento dell'abate di Lucullano parte dal nucleo del Vangelo che è l'amore e, da questa esperienza redentiva del rapporto con il Signore, la creatura riceve "grazia su grazia" (Gv 1,16) e inizia il suo "santo viaggio" (Sal 83,6) nella via perfectionis, che è via humilitatis e, insieme irriducibilmente, via caritatis.

Senza carità non vi è umiltà e senza umiltà non vi è carità, pare l'appello di Eugippio. Egli, inoltre, mai fa cenno nell'opera a differenze tra i monaci, che siano essi chierici o laici, così come esplicitamente non si trova menzione dei sacramenti. Eppure si intende bene che essi sono il vissuto di cui la comunità si nutre, colonne e fondamento di quella reciprocità fraterna che emana dalla comunione eucaristica e dalla misericordia della riconciliazione con $\mathrm{Dio}^{70}$.

Ciò significa che l'uomo di Dio, e più semplicemente il cristiano in sé, è comunque sempre tenuto al „comandamento nuovo" del Vangelo. Il monaco ne è un chiamato "più consapevole", forse privilegiato per contesto, ma non più "speciale", e di questa "consapevolezza" del molto ricevuto Eugippio si fa servitore ed educatore. La sua umiltà non fa valere in ciò neppure il fatto che egli stesso fosse presbitero.

Tutti sono eletti dalla misericordia di Dio nella regola d'oro del Vangelo, che non fu rivolta ad alcuna categoria, classe o casta particolare di persone: l'amore a Dio e al prossimo è regola universale di riscatto e di convivenza, di sapienza e di civiltà. Nessuno deve considerarsi superiore ad altri e ognuno è tenuto a lasciarsi condurre dallo Spirito Santo nella conversione e nella plasmazione di questa verità che si riceve unicamente dall'“alto".

$$
* * *
$$

70 Cf. Degórski - Mirri, Eugippio. La Regola, p. 41-42. 
L'attualità di un testo monastico così ponderato per saggezza umana e così esigente nella perfetta sintonia con la sapienza evangelica è innegabile. Il respiro ecumenico travalica la tradizione orientale e occidentale della Chiesa: è una Regula che parla al cuore dell'uomo per offrirgli 1" "istruzione" della "pace vera", "non come la dà il mondo" (Gv 14,27), perché è quella riposta nel primato della carità di Cristo.

Il Vangelo viene qui ripresentato come forza prorompente nella storia e nella persona. La carità è per Eugippio la pedagogia che esso inaugura per educare interiormente l'uomo responsabilizzandolo. La sua potenza, infatti, lo libera da se stesso, perché lo porta verso l'Altissimo e verso il prossimo. Il resto lo compie la paternità di Dio, per i meriti della Redenzione del Figlio suo e per il dono santificante dello Spirito Paraclito, il quale "guiderà alla verità tutta intera" (Gv 16,13).

\section{The Sources and Theological Themes of the Regula of Eugippius}

(summary)

The article demonstrates the following sources of Eugippius" "Rule" and discusses them: St. Augustine of Hippo (the Ordo monasterii and the Praeceptum); the "Rules": the Regula quattuor Patrum and the Regula Magistri; St. Basil the Great (the Regula [a Rufino latine versa]); St. Pachomius the Great (the Regula); Novatus Catholicus (the Sententia de humilitate et oboedientia et de calcanda superbia); St. John Cassian (the Conlationes Patrum and the De institutis coenobiorum); St. Jerome (the Epistula 125, 9). In its second part, the article discusses the theological subject matter of the Regula of Eugippius, also pointing to the timeliness of its message and its relevance to our times.

Keywords: Eugippius; the Regula Eugippii; Monasticism; Spirituality

\section{Źródla i tematyka teologiczna Reguly Eugipiusza}

(streszczenie)

Artykuł wyodrębnia następujące źródła Reguły Eugipiusza oraz je omawia: św. Augustyn z Hippony (Ordo monasterii oraz Praeceptum); reguły anonimowe: Regula quattuor Patrum oraz Regula Magistri; św. Bazyli Wielki (Regula [a Rufino latine versa]); św. Pachomiusz (Regula); Novatus Catholicus (Sententia de humilitate et oboedientia et de calcanda superbia); św. Jan Kasjan (Conlationes Patrum oraz De institutis coeno- 
biorum); św. Hieronim ze Strydonu (Epistula 125, 9). W swej drugiej części artykuł omawia tematykę teologiczną Reguły Eugipiusza, wskazując także na aktualność jej przesłania również i w dzisiejszych czasach.

Słowa kluczowe: Eugipiusz; Regula Eugippii; monastycyzm; duchowość

\section{Bibliografia}

\section{Fonti}

Augustinus Hipponensis, Regula, in: La Règle de saint Augustin, ed. L. Verheijen, Collection des Études Augustiniennes, Paris 1967.

Basilius Caesariensis, Regula [a Rufino latine versa], CSEL 86.

Cassiodorus, De institutione divinarum et saecularium litterarum, in: Cassiodor, Institutiones divinarum et saecularum litterarum, ed. W. Bürsgens, Freiburg 2003.

Eugippius, Epistula ad Probam, CSEL 9/1, 1-4.

Eugippius, Regula, in: Eugipii Regula, ed. F. Villegas - A. de Vogüé, CSEL 87, Vindobonae 1976.

Eugippius, Vita S. Severini, CSEL 9/2 e SCh 374.

Ferrandus Carthaginensis, Epistulae et opuscula, PL 67, 887-950.

Fulgentius Ruspensis, Epistulae, SCh 487.

Hieronymus Stridonensis, Epistulae, CSEL 56.

Iohannes Cassianus, Conlationes Patrum, PL 49, 477-1321; CSEL 13; SCh 42, SCh 54, SCh 64.

Iohannes Cassianus, De institutis coenobiorum, PL 49, 43-476; CSEL 17, 3-231; SCh 109.

Isidorus Hispalensis, De viris illustribus, PL 83, 1081-1106.

Novatus Catholicus, Sententia de humilitate et oboedientia et de calcanda superbia, PL 18, 67-70; PLS 1, 672 e 1751; ed. F. Villegas, RBen 86 (1976).

Pachomius, Regula, in: Pachomiana Latina, ed. A. Boon, Bibliothèque de la Revue d'Histoire Ecclésiastique 7, Louvain 1932.

Regula Magistri, SCh 105-106.

Regula quattuor Patrum, ed. J. Neufville, RBen 77 (1967) p. 72-91. 


\section{Studi}

Bozzi M. - Grilli A., Introduzione, in: Regola del Maestro, v. 1, ed. M. Bozzi - A. Grilli, Brescia 1995, p. 13-27.

Buedinger M., Eugippius. Eine Untersuchung, "Sitzungsberichte der Österreichischen Akademie der Wissenschaften in Wien" 91 (1978) p. 793-814.

Cappuyns M., Eugippius, DHGE XV 1376-1378.

Degórski B., I Libri sapienziali nella letteratura monastica delle origini (secc. IV-V), in: Letture cristiane dei Libri Sapienziali, SEA 37, Roma 1992, p. 65-86.

Degórski B., L'elenco delle citazioni dei Libri sapienziali nella letteratura monastica del IV e V secolo, RT 40 (1993) p. 119-154.

Degórski B., Il primissimo monachesimo nell'Africa Latina, VoxP 53-54 (2009) p. 591-598.

Degórski B., Św. Augustyn z Hippony - krzewiciel monastycyzmu w Afryce Łacińskiej, in: W pedagogicznej stużbie narodów, ed. M. Włosiński, Włocławek 2009, p. 123-133.

Degórski B., De Sancto Aurelio Augustino Episcopo Hipponensi veritatis Christianae defensore eximio, in: Nauka o wymiarach prawdy. Studio sulle valenze della verità. Rozmery pravdy. Księga Pamiątkowa z okazji 70. Rocznicy Urodzin ks. Profesora Mariana Włosińskiego, ed. S. Kunikowski, Włocławek 2015, p. 103-115.

Degórski B. - Mirri L., Eugippio. La Regola, Collana di Testi Patristici 183, Roma 2005.

Degórski B. - Mirri L.M., Eugippio Abate. La Regola, in: A. Genovese, Eugippio Abate. Opere, Scrittori della Chiesa di Aquileia 7, Roma - Aquileia 2012, p. 139-249.

Genestout A., Le plus ancien témoin manuscrit de la Règle du Maître: le Parisinus latin 12634, "Scriptorium” 1 (1946/47) p. 129-142.

Genovese A., "Velut ex ingenti prato floribus asperso caelestibus". Gli Estratti dalle Opere di Sant'Agostino di Eugippio, "Urbaniana University Journal” 70/3 (2017) p. 177-215.

Gobry I., Storia del monachesimo, v. 1-2, Roma 1991.

Gorman M.M., The Manuscript Tradition of Eugippius, "Excerpta ex operibus sancti Augustini”, RBen 92 (1982) p. 7-32, 229-265.

Häussling A.A., Das Commemoratorium des Eugippius und die Regula Magistri und Regula Benedicti, in: Regulae Benedicti Studia, ed. B. Jaspert - E. Manning, Annuarium Internationale 5, Hildesheim 1977, p. 33-42. 
Hofmann J., Das Werk des Abtes Eugippius. Zum literarischen Vermächtnis eines spätantiken Augustinus-Kenners an die frühmittelalterliche Kirche des Abendlandes, ZKG 109 (1998) p. 293-305.

Kardong T., Justitia in the Rule of Benedict, SMon 24 (1982) p. 43-73.

Krausgruber M., Die Regel des Eugippius. Die Klosterordnung des Verfassers der Vita Sancti Severini im Lichte ihrer Quellen. Text, Übersetzung und Kommentar, Innsbruck 1996.

Lawless G., An Overview of Augustine's Monasticism and some Suggestions for Further Research, in: The Spirituality of Ancient Monasticism. Acts of the International Colloquium held in Cracow-Tyniec 16-19 $9^{\text {th }}$ November 1994. Specialized Contributions, ed. M. Starowieyski, Pontificia Academia Theologica Cracoviensis, Facultas Theologica. Studia 4/1, Tyniec - Kraków 1995, p. 135-161.

Leclercq H., Eugippius, DACL V 702-704.

Leclercq J., Autor d'un manuscrit de la Règle du Maître, RBen 57 (1947) p. 210-212.

Leyser C., Shoring fragments against ruin? Eugippius and the six-century of the florilegium, in: Eugippius und Severin. Der Autor, der Text und der Heilige, ed. W. Pohl - M. Diesenberger, Wien 2001, p. 68-70.

Noll R., Eugippius. Das Leben des heiligen Severin, Berlin 1963.

Obrycki K., Wstęp, in: Eugipiusz. Żywot Św. Seweryna. Reguła, ed. K. Obrycki, ŹM 13, Tyniec - Kraków 1996, p. 38-44.

Penco G., La composizione sociale delle comunità monastiche nei primi secoli, SMon 4 (1962) p. 257-281.

Penco G., Sul concetto del monastero come “schola”, CCist 32 (1970) p. 329-333.

Plinval G. de, Eugippius, DSp IV 1684-1685.

Pricoco S., Introduzione, in: La regola di S. Benedetto. Le regole dei Padri, ed. S. Pricoco, Milano 1995, p. IX-XVII.

Régerat Ph., Introduction, in Eugippe. Vie de Saint Séverin, ed. Ph. Régerat, SCh 374, Paris 1991, p. 122-135.

Sanchis D., Pauvreté monastique et Charité fraternelle chez saint Augustin, "Augustiniana" 8 (1958) p. 5-21.

Siniscalco P., Il numero primitivo degli Excerpta di Eugippius, REAug 10 (1964) p. 331-342. 
Storia della Chiesa, ed. H. Jedin, Già e non ancora 3, Milano 1988.

Tamburrino P., La Regula Magistri e l'origine del potere abbaziale, CCist 28 (1966) p. $160-173$.

Turbessi G., Regole monastiche antiche, Roma 1978.

Villegas F. - Vogüé A. de, Praefatio, in: Eugippii Regula, CSEL 87, Vindobonae 1976, p. VII-XX.

Vogüé A. de, Nouveaux aperçus sur une règle monastique du VI siècle, RAM 41 (1965) p. 19-29.

Vogüé A. de, La Règle d'Eugippe retrovée?, RAM 47 (1971) p. 233-265.

Vogüé A. de, Sub Regula uel Abbate, in: Rule and Life. An Interdisciplinary Symposium, Massachusetts 1971, p. 21-63.

Vogüé A. de, Les conseils évangéliques chez le maître et saint Benoît, in: Los consejos evangélicos en la tradición monástica, Abadia de Silos 1975, p. 13-27.

Vogüé A. de, Quelques observations nouvelles sur la Règle d'Eugippe, "Benedictina" 22 (1975) p. 31-41.

Vogüé A. de, Cassien, le Maître et Benoît, in: Commandements du Seigneur et libération évangélique, Roma 1977, p. 223-235.

Vogüé A. de, De Cassien au Maître: le titre du chapitre de l'humilté, SMon 24 (1982) p. 247-261. Vogüé A. de, Novati Sententia de Humilitate et Obedientia, DIP VI 441-442.

Vogüé A. de, Regula Eugippii, DIP VII 1575-1576.

Vogüé A. de, L'horaire de l'Ordo Monasterii. Ses rapports avec le monachism égyptien, in: Homo Spiritalis, Würzburg 1987, p. 240-258.

Vogüé A. de, L'influence du monachisme des Kellia en Occident, in: The Spirituality of Ancient Monasticism. Acts of the International Colloquium held in Cracow-Tyniec 16-19 $9^{\text {th }}$ November 1994. Specialized Contributions, ed. M. Starowieyski, Pontificia Academia Theologica Cracoviensis, Facultas Theologica. Studia 4/1, Tyniec Kraków 1995, p. 164-168.

Vogüé A. de, L'influenza di Basilio sul monachesimo occidentale, in: Basilio tra Oriente e Occidente. Convegno Internazionale "Basilio il Grande e il Monachesimo Orientale”, Cappadocia, 5-7 ottobre 1999, Comunità di Bose, ed. S. Brock - M. Cortesi - A. de Vogüé - J.-R. Pouchet, Magnano 2001, p. 209-224. 